\title{
Metastasiertes Kolorektalkarzinom
}

\section{Zulassung für humanen EGFR-Antikörper erweitert}

\author{
Panitumumab, ein humaner Antikörper gegen den epidermalen \\ Wachstumsfaktorrezeptor (EGFR), ist nun auch kombiniert mit \\ Oxaliplatin- und Irinotecan-haltigen Chemotherapien bei Pati- \\ enten mit metastasiertem kolorektalem Karzinom (mCRC) und \\ nicht mutiertem K-Ras einsetzbar.
}

Die Europäische Kommission hat nach Angaben von Amgen einer Variation der bedingten Zulassung von Panitumumab (Vectibix ${ }^{\circ}$ ) zugestimmt. Jetzt ist der Antikörper gegen EGFR in Kombination mit FOLFOX (Folinsäure, 5-Fluorouracil [5-FU], Oxaliplatin) indiziert für die Erstlinientherapie von mCRCPatienten, deren Tumoren EGFR und das Proto-Onkogen K-Ras im Wildtyp exprimieren. Zusätzlich können diese Patienten Panitumumab gemeinsam mit FOLFI-
RI (Folinsäure, 5-FU, Irinotecan) erhalten, wenn zuvor eine Fluoropyrimidin-basierte Chemotherapie (außer Irinotecan) versagt hatte. Die Zulassungserweiterung basiert auf den Ergebnissen der PRIME-Studie zur Erstlinientherapie [Douillard J et al. J Clin Oncol. 2011; 9(suppl; abstr 3510)] und der Phase-III-Studie '181 zur Zweitlinientherapie [Peeters $\mathrm{M}$ et al. J Clin Oncol. 2010;28:4706-13]. Jean-Yves Douillard, Nantes, Frankreich, betonte, dass allerdings nur mCRC-Patienten mit
K-Ras-Wildtyp ein PanitumumabRegime erhalten sollten. In der PRIME-Studie hatte die Gabe des EGFR-Antikörpers sogar einen nachteiligen Effekt, verabreichte man ihn in der Erstlinie gemeinsam mit FOLFOX4 an Patienten mit mutiertem K-Ras: Das Progressionsrisiko erhöhte sich um $27 \%$ verglichen mit der alleinigen Chemotherapie (HR 1,27; 95\%-KI $1,04-1,55 ; \mathrm{p}=0,02)$. Patienten mit Wildtyp-K-Ras lebten dagegen unter der Kombination 1,6 Monate länger progressionsfrei als unter FOLFOX4 allein (10 vs. 8,6 Monate; $\mathrm{p}=0,01$ ).

Media Roundtable im Rahmen des European Multidisciplinary Cancer Congress am 25. September 2011 in Stockholm; Veranstalter: Amgen

Kurz notiert

\section{Aktuelle Strategien gegen Meningeosis neoplastica}

Das Auftreten einer Meningeosis neoplastica ist bei Lymphomen und Leukämien sowohl bei Primärdiagnose als auch im Rezidiv mit einer ungünstigen Prognose und einem schlechten Behandlungsergebnis der Grunderkrankung verbunden. Dieter Hoelzer, Frankfurt am Main, erläutert in einem Interview die aktuellen Strategien gegen Meningeosis neoplastica und gibt einen Einblick in aktuelle Forschungsprojekte mit liposomalem Cytarabin (DepoCyte ${ }^{\oplus}$ ). Diese spezielle galenische Formulierung des bewährten Zytostatikums Cytarabin verfügt über eine lange Plasmahalbwertszeit von zwei Wochen und verteilt sich über diesen Zeitraum gleichmäßig im Liquor. DepoCyte ${ }^{\oplus}$ muss daher nur einmal in zwei Wochen intrathekal appliziert werden. Das Interview steht online auf der Webseite der Deutschen Krebsgesellschaft unter http://www.krebsgesellschaft.de/index.php?seite=arzt_2011_all_lymphome_zns_manifestation\&navigation $=88734$ \&system_id=\&kanal=html db Nach Informationen von Mundipharma
Eisencarboxymaltose bei tumorbedingtem Eisenmangel

Seit Oktober 2011 ist der dextranfreie Eisencarboxymaltosekomplex Ferinject ${ }^{\oplus}$ als $1.000-\mathrm{mg}$ Packung ( 2 x 10ml; PZN 8805803) erhältlich. Das Medikament wird als Kurzinfusion oder Injektion zur Behandlung von Eisenmangel verabreicht, wenn eine orale Eisentherapie nicht wirksam oder durchführbar ist. Dies betrifft z. B. Patienten mit tumorbedingter Anämie, die häufig durch funktionellen Eisenmangel verursacht wird. Funktioneller Eisenmangel geht mit einer Störung der intestinalen Eisenresorption und einer reduzierten Mobilisierung des Eisens aus den Speichern einher. Eine Transferrinsättigung (TSAT) von $<20 \%$ ist ein wichtiger diagnostischer Marker für einen funktionellen Eisenmangel. Die angemessene Gesamtdosis des Medikaments muss für jeden Patienten individuell berechnet werden und darf nicht überschritten werden. An Nebenwirkungen sind u. a. Kopfschmerzen, Übelkeit, Verstopfung, Durchfall, und ein Anstieg des Leberenzyms Alaninaminotransferase zu beachten. sg
5-Jahres-Nachuntersuchung der VISTAStudie: Überlebensvorteil mit VMP

Die Ergebnisse der 5-Jahres-Nachuntersuchung der VISTA-Studie bestätigen den deutlichen Überlebensvorteil durch die Bortezomib-haltige Therapie [San Migeul J et al. ASH 2011; San Diego; Abstract \#476]. In der VISTA-Studie waren 682 nicht vorbehandelte Patienten mit multiplem Myelom, die nicht für eine Hochdosis-Chemotherapie mit anschließender Stammzelltransplantation geeignet waren, entweder mit VMP (Bortezomib plus Melphalan/Prednison) oder nur mit MP (Melphalan/Prednison) behandelt worden. Mit VMP wurde eine Gesamtüberlebensrate von 56,4 Monaten (vs. 43,1 Monate unter MP Monate) erreicht. Dieser signifikante Überlebensvorteil von 13,3 Monaten ( $p=$ 0,0004) entspricht einem $31 \%$ niedrigeren Mortalitätsrisiko. Ähnliche Ergebnisse ließen sich bisher nur mit hochdosierter Chemotherapie und autologer Stammzelltransplantation erreichen, für die aber ein großer Teil der Patienten nicht infrage kommt. $\mathrm{db}$ 